March 26, 2002

\title{
On the Geometrical Character of Gravitation
}

\author{
Chryssomalis Chryssomalakos and Daniel Sudarsky \\ Instituto de Ciencias Nucleares \\ Universidad Nacional Autónoma de México \\ Apdo. Postal 70-543, 04510 México, D.F., MEXICO \\ chryss@nuclecu.unam.mx, sudarsky@nuclecu.unam.mx
}

\begin{abstract}
The issue of whether some manifestations of gravitation in the quantum domain, are indicative or not of a non-geometrical aspect in gravitation is discussed. We examine some examples that have been considered in this context, providing a critical analysis of previous interpretations. The analysis of these examples is illustrative about certain details in the interpretation of quantum mechanics. We conclude that there are, at this time, no indications of such departure from the geometrical character of gravitation.
\end{abstract}




\section{Contents}

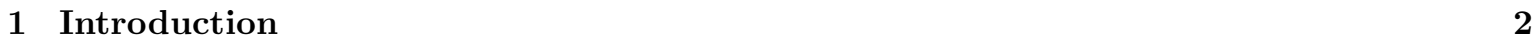

2 Geometry and Gravitation 2

\begin{tabular}{|lll}
\hline The COW Experiment & 4
\end{tabular}

4 The Neutrino Oscillation Clocks

\begin{tabular}{|lrr}
5 & Discussion & 9
\end{tabular}

\section{Introduction}

Gravitation is the only one of the known forces for which there is at present no fully satisfactory quantum description, despite the recent progress that some of the approaches to the problem have achieved. At some point, workers in the field even wondered whether gravity should be quantized at all or whether it should, instead, be considered as an effective phenomenon, of the type described, for example, by thermodynamics [5] — in this latter case it would be clearly inappropriate to attempt a description at the quantum level. Fundamental considerations naturally enter in the analysis of such questions. For instance, if gravity could be thought to be classical in all situations, then one could use the gravitational field associated with a particle to determine its location and state of motion to an accuracy higher that that allowed by the uncertainty principle. Another line of attack involves experiments that probe some aspects of gravitation at the quantum level. Although recent ideas are suggestive of possible experimental indications of quantum gravitational features [2], the aspect that has in fact been subject to experiment is the opposite end of the problem, namely, the effect of gravitation on the behavior of quantum systems. The most famous among these tests is the COW [3] experiment, in which a gravity-induced quantum phase difference is measured by the interference of two neutron beams that travel through regions of different gravitational potential. This remarkable confirmation of some of the basic assumptions inherent to quantum mechanics and to gravitation led to a whole series of other related experiments, either real (see, e.g. [4] and references therein) or gedanken [1]. The hope behind all these efforts is to draw lessons about the behavior of gravity in the quantum realm.

One issue that takes center stage in these discussions is whether gravity maintains its geometrical nature at the quantum level. For instance, a well-known quantum mechanics textbook [6] asserts that the outcome of the COW experiment is indicative of a non-geometric aspect of gravitation, since the effect measured is sensitive to the mass of the particles used. A more puzzling case is the gedanken use of neutrino oscillations (between different mass eigenstates) to construct clocks that do not "red-shift" in a universal way under the influence of gravitational fields [1]. The aim of this paper is to provide a well defined set of criteria in order to determine whether particular gravitational effects can be said to be of a non-geometrical nature, and to examine in this light the situations that have been thought to reflect such non-geometrical aspects.

\section{Geometry and Gravitation}

The basic idea behind the geometrical description of gravity is the equivalence principle (E.P.), which leads to the possibility of constructing effective locally inertial frames (LIFs) about every point in space-time. In practice, the construction itself of LIFs is tied to the EP, given the fact that it is 
impossible to isolate any particle from the influence of gravity and thus to obtain any object that can be considered free of interactions, for which the law of inertia might have meaning. Therefore, the EP is not to be regarded as a circumstantial feature of the gravitational interaction, but as part of the foundations of physics itself.

The geometrical character of gravitation then is tied with the possibility of simulating gravitation by using an accelerated frame. Equivalently, one can "cancel" gravitation by accelerating without constraints under the influence of gravity, i.e., by going to a freely falling frame mentioned in the introduction measure, in one way or another, quantum effects in the presence of a gravitational field. In view of the above discussion, our general strategy will be to look at these experiments from a LIF and compare the results with the case of zero gravitational field.

Let us start by being more precise with our notion of geometricity of gravitation. We must clarify that we seek a phenomenological definition, appropriate for examining both real and gedanken experiments, and not a theoretical one, such as the one given in terms of the requirement of general covariance [7]. The latter, although both appropriate and useful when considering a given classical theory, would be neither when applied to the analysis of certain experimental situations, in particular those pertaining to the quantum domain, e.g., in the possible quantum gravity induced modifications of the dispersion relations of photons [2].

One can start by saying simply that

D1 Gravity is geometrical if all its effects can be locally canceled (or simulated) by a suitable choice of the reference frame in which their description takes place.

One needs to be careful with the above statement. For one, care is needed in treating the notion of locality, even at the classical level, when dealing, for instance, with particles interacting with electromagnetic fields. Second, one can imagine experimental devices designed to measure some components of the Riemman tensor, e.g., a differential accelerometer, the behavior of which in a curved spacetime could never be simulated in a flat one. Third, one should take into account the possibility that there might exist new kinds of matter 7 with nonminimal coupling to gravitation, which, just like a differential accelerometer, would behave differently in a flat than in a curved spacetime, but the existence of which would in no way put in question the geometrical character of gravitation. Finally, one should note that the above definition, natural as it may be in a classical context, can in principle become suspicious when applied to quantum systems, because of the quantum limits on localizability of the latter.

In view of the above discussion, a general formal definition of what is to be understood by the geometricity of gravitation will not be attemted here. Rather, we focus on a set of useful criteria, that permit the analysis of the experiments mentioned in the introduction. As a first step, we concentrate on the propagation of a "free particle", the latter being defined as any particle (including, e.g., atoms and subatomic particles) with no electric charge (or higher multipole moments), separated from other particles by distances that ensure that the nuclear forces are not operative. We propose the following definition

D2 Gravitation is geometrical in nature, if the description of the propagation of free particles given by their wave function, in a given gravitational field, is identical to the one obtained in an appropriately moving frame in Minkowski spacetime.

Some qualifications are necessary. First, the description mentioned above should refer to a region of spacetime which is large compared with that over which the particle's wavefunction is appreciable.

\footnotetext{
${ }^{1}$ Notice that going to a "freely falling frame" involves, in general, an appropriate state of rotation, in addition to an appropriate state of acceleration.

${ }^{2}$ We have in mind particles associated with supersymmetry or matter described by the cosmological fields (quintessence and the like) that have been considered as candidates for the "dark energy".
} 
At the same time, it should be small compared with the region over which tidal effects become appreciable. Finally, any discrepancies should scale in an appropriate way (which depends on the actual quantity being measured) with the size of the region in question. In fact, with such corrections it should be possible to determine the Riemann tensor in the above region.

We can now deal with more complex systems, such as devices sensitive to tidal forces, or matter with nonminimal couplings. In those cases the geometrical character of gravitation will be tied to the notion that all of the local effects of gravitation should be accounted for by the Riemann tensor, and in particular, that if the Riemann tensor vanishes in a region, all experiments carried out completely within that region should be exactly reproducible in an appropriately moving frame in flat spacetime.

As an example, we apply the above concepts to the following statement

S1 The geometrical nature of gravity requires that all clocks red-shift in a universal way under its influence.

Imagine a (poorly designed) clock, sensitive to second derivatives of the gravitational potential (i.e., tidal forces). Such a clock does not red-shift in the same way as, say, an atomic clock and, therefore, (Si] would lead us to conclude that gravity in not geometrical in nature. On the other hand, our criterion (D2) provides for discrepancies between the two clocks that scale appropriately with the size of the clocks. Clearly, the difference in red-shifts between the two clocks falls within this provision, and therefore, (SE) is incompatible with (D/2). We will say accordingly that (S1) is incorrect.

Having said all this we proceed to examine a number of experiments which, it has been argued, indicate non-geometrical aspects of gravitation. We will show that these interpretations are not appropriate, in the sense advocated earlier. Actually, given the fact that the experiments do not involve tidal effects, it is enough to consider them in light of (D[1), even though in principle, and given the quantum nature of the probes, one should rely on the more refined definition (D2). We will refer, in the discussion of the last section, to a new class of possible experiments, the analysis of which would rely in an essential way on (D2).

As a final point, we would like to stress that even though one is, of course, free to use a different definition of "the geometrical character of gravitation", we believe that the notion expressed by (D2) is the closest in spirit to the general relativistic one, as applied in the realm of classical physics, and is such that one can expect it to be appropriate for the quantum domain.

\section{The COW Experiment}

Next we turn to this famous neutron intereferometry experiment, in which one considers the interference pattern of two neutron beams that travel on two paths on a plane. The observational quantities are related to change in this interference pattern when the plane is rotated in such a way that, at one moment the plane is perpendicular to the gradient of the earth's gravitational potential, and at some other instant it is tangent to it. In this way one measures the dependence of the phase difference in the two neutron beams on the gravitational field of the earth. The result turns out to depend on the neutron's mass, and this has lead to interpretations of this experiment as showing a non geometrical aspect of gravity. Underlying such interpretations there is a notion that

\section{S2 The geometrical nature of gravitation should make it impossible to determine the mass of a particle through the use of purely passive gravitational effects.}

Here the problem is due to the failure to recognize that, at the quantum level, the mass of a particle is associated with a geometrical scale. In fact we can determine, even in the absence of gravity, the mass of a particle by purely geometrical means, i.e., relying only on the behavior of freely propagating particles and not on properties of their interactions: take a monochromatic particle beam and measure 


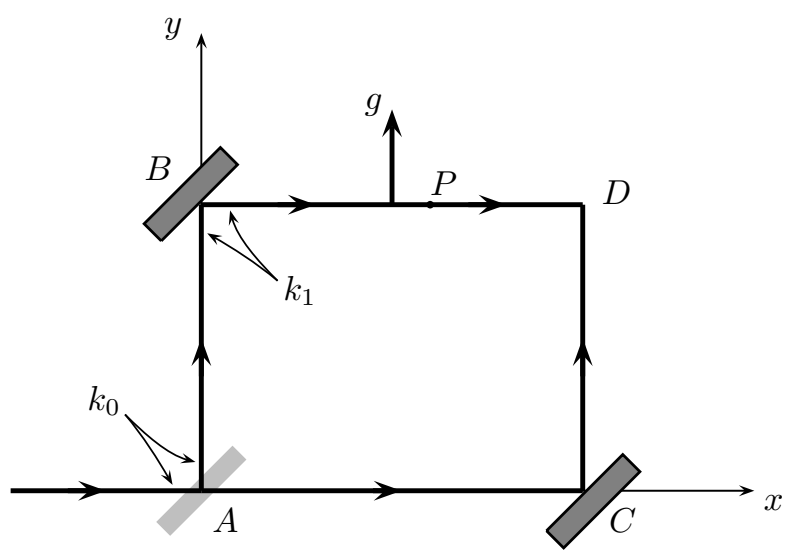

Figure 1: The COW experiment, as seen from an inertial frame. The wave at $B$ was split at $A$ when $A$ was moving downwards with velocity $g a / v_{0}$. The wave vector is Doppler red-shifted, $k_{1}=k_{0}(1-\epsilon)$, $\epsilon \equiv g a / v_{0}^{2}$. The transition from $k_{0}$ at $A$ to $k_{1}$ at $B$ is linear in the height $y$. The wave at $P$ was reflected at $B$ when $B$ was moving downwards with velocity $g x_{P} / v_{1}=g x_{P} / v_{0}+\mathcal{O}\left(\epsilon^{2}\right)$. The resulting Doppler blue-shift cancels the $x$-dependent part of the red-shift at $A$ and makes the wave vector constant along $B D$. The contributions to the phase from $A B$ and $C D$ cancel, so that $\Delta \phi=\left(k_{0}-k_{1}\right) b=\epsilon k_{0} b$.

its momentum $p$ by a simple double slit experiment. Then make the double slit experimental set-up move with velocity $V$ with respect to the laboratory, and measure the momentum $p^{\prime}$ of the beam in the new frame - the mass is read off as $M=\frac{p^{\prime}-p}{V}$. Let us apply our criterion (D2): the dimensions of the apparatus used are much smaler than the scale over which it could detect tidal effects, and the neutron wavelength is much smaller than that. Thus, we should be able to account for the experimental result from the point of view of an inertial observer who watches the entire apparatus moving upwards with constant acceleration $g$. The authors of [3] allude to such a description — we give a short outline of ours for completeness.

Referring to Fig. 1, suppose that the apparatus is accelerating upwards and has, momentarily, zero velocity. Then the wave vector of the beam at the lower part of the segment $A B$, right above $A$, is equal to the incoming value $k_{0}$. Further up that segment, for a fixed time $t$, the wave vector decreases linearly with the height because of Doppler shift (when it was emitted by the beam splitter at $A$, the latter was moving downwards). The wave on the horizontal segment $B D$, immediately to the right of $B$, suffered no Doppler shift when it was reflected at $B$ because, by assumption, $B$ is momentarily at rest. One might think that at points further to the right on $B D$ the wave vector will keep decreasing but this is not so: when the wave at, say, point $P$ was reflected by the mirror at $B$, the latter was moving downwards with a velocity that is bigger the further to the right $P$ is. A simple calculation shows that the Doppler shift suffered in this second reflection cancels the $x$-dependence of the wave vector. Similar remarks apply to the $A C D$ path and the calculation of the resulting phase difference at $D$ is algebraically identical with the one in the presence of gravity. Thus, according to (D2), the COW experiment supports rather than negates the geometrical nature of gravitation and (S2) is therefore incorrect.

\section{The Neutrino Oscillation Clocks}

Recently, a gedanken experiment has been considered, in which two clocks that base their operation on neutrino oscillations "red-shift" differently due to the effects of gravitation [1]. This has been interpreted as an indication of a non-geometrical aspect of gravitation. We will examine this assertion using the criterion set forth in (D2). 
The set-up uses the Lense-Thirring effect — we avoid inessential complications by considering a simplified setting in which this is the only relevant gravitational effect present. Consider a hollow spherical shell with mass $M$ and radius $R(R>>M G)$, rotating about the $z$-axis with angular velocity $\vec{\alpha}$. We have, besides the constant gravitational potential $\Phi_{0}=-G M / R$ (relative to points at infinity), a gravitomagnetic field $\vec{B}$, given by $\vec{B}=\frac{2 G M}{3 R} \vec{\alpha}$. The spacetime metric inside the rotating shell, in first order perturbation theory (with both $\Phi_{0}$ and $B$ of first order), is

$$
d S^{2}=\left[-\left(1-2 \Phi_{0}\right) d t^{2}+d x^{2}+d y^{2}+d z^{2}\right]+2 B(y d x-x d y) d t
$$

where $B=|\vec{B}|$. One now constructs two clocks, $I$ and $I I$, that base their operation on oscillations between suitable superpositions of the mass and spin eigenstates $\mid m_{i}, \hat{z} \pm>\left(m_{1}, m_{2}\right.$ are the mass eigenvalues and $\hat{z} \pm$ refer to the $S_{z}$ eigenstates - we will assume $m_{1}>m_{2}$ ). Clock $I$ oscillates between the states

$$
\begin{aligned}
\left|Q_{a}\right\rangle & \equiv \frac{1}{\sqrt{2}}\left|m_{1}, \hat{z}+\right\rangle+\frac{1}{\sqrt{2}}\left|m_{2}, \hat{z}+\right\rangle \\
\left|Q_{b}\right\rangle & \equiv-\frac{1}{\sqrt{2}}\left|m_{1}, \hat{z}+\right\rangle+\frac{1}{\sqrt{2}}\left|m_{2}, \hat{z}+\right\rangle,
\end{aligned}
$$

while clock $I I$ uses the pair

$$
\begin{aligned}
\left|Q_{A}\right\rangle & \equiv \frac{1}{\sqrt{2}}\left|m_{1}, \hat{z}+\right\rangle+\frac{1}{\sqrt{2}}\left|m_{2}, \hat{z}-\right\rangle \\
\left|Q_{B}\right\rangle & \equiv-\frac{1}{\sqrt{2}}\left|m_{1}, \hat{z}+\right\rangle+\frac{1}{\sqrt{2}}\left|m_{2}, \hat{z}-\right\rangle .
\end{aligned}
$$

In other words, in the four-dimensional state space available, with basis $\left\{\left|m_{1}, \hat{z}+\right\rangle,\left|m_{1}, \hat{z}-\right\rangle,\left|m_{2}, \hat{z}+\right\rangle\right.$, $\left.\left|m_{2}, \hat{z}-\right\rangle\right\}$, each clock works in a two-dimensional subspace. That the time evolution of the clocks does not lead outside of this subspace will be obvious, since the states appearing in the r.h.s. of (2), (3), will be eigenstates of all hamiltonians considered in the sequel. Clock $I$ runs by monitoring the transition $a \rightarrow b$, which occurs with probability $P(a \rightarrow b)$. For example, an ensemble of particles oscillating between $\left|Q_{a}\right\rangle$ and $\left|Q_{b}\right\rangle$ could be observed, with clock $I$ "ticking" every time a maximum in the population of the state $\left|Q_{a}\right\rangle$ is observed - similar remarks apply to clock $I I$.

In the absence of any gravitational field the time-evolution of the clocks is described by the Hamiltonian

$$
\mathbf{H}_{0}=\mathbf{m} c^{2},
$$

where $\mathbf{m}$ is the mass operator and we neglect the kinetic term. In this case, the spin degree of freedom is "spectator" and the two clocks tick with the same frequency $2 \omega_{0}=\omega_{I}=\omega_{I I}$, where

$$
\omega_{0}=\frac{\left(m_{1}-m_{2}\right) c^{2}}{2 \hbar} \equiv \frac{\Delta m c^{2}}{2 \hbar} .
$$

Now we place the clocks inside the rotating shell, where the Hamiltonian is

$$
\mathbf{H}^{\prime}=\mathbf{m} c^{2}\left(1-2 \Phi_{0}\right)+\overrightarrow{\mathbf{S}} \cdot \vec{B}
$$

( $\overrightarrow{\mathbf{S}}$ is the particle spin operator). For clock $I$ the spin is still "spectator" and its frequency is simply multiplied by the factor $\lambda \equiv 1-2 \Phi_{0}$,

$$
\omega_{I}^{\prime}=\lambda \omega_{I}
$$

\footnotetext{
${ }^{3}$ We have set, for simplicity, the "mixing angle" $\theta$ of $\frac{1}{1}$ equal to $\pi / 4$ - the results do not depend essentially on this choice.

${ }^{4}$ One gets $P_{a \rightarrow b}=\sin ^{2} \omega_{0} t=\left(1-\cos 2 \omega_{0} t\right) / 2=P_{A \rightarrow B}$.
} 
Clock $I I$, on the other hand, receives an additional shift in its frequency by the energy difference of the two spin eigenstates that enter in $\left|Q_{A}\right\rangle,\left|Q_{B}\right\rangle$,

$$
\omega_{I I}^{\prime}=\lambda \omega_{I I}+B
$$

Does this effect indicate a non-geometric aspect of gravity? Let us apply again the criterion (D2). To start with, the particle system is assigned a length scale of order $1 / m$, which we consider fixed once and for all. The gravitational field has a length scale given by $R$, but the gravitomagnetic field inside the shell can be kept constant while scaling both $M$ and $R$ simultaneously upward until $R>>1 / m$. Thus the effect remains unchanged when we arrange the scales to satisfy the requirements of (D2). The issue is then, does the effect persist when one moves to an inertial reference frame, the latter being defined as one where the metric becomes locally Lorentzian?

Going to a (primed) frame rotating with an arbitrary angular velocity $\Omega \hat{z}$,

$$
t=\left(1+\Phi_{0}\right) t^{\prime}, \quad x=x^{\prime} \cos \Omega t^{\prime}-y^{\prime} \sin \Omega t^{\prime}, \quad y=y^{\prime} \cos \Omega t^{\prime}+x^{\prime} \sin \Omega t^{\prime}, \quad z=z^{\prime},
$$

the metric of (11) becomes

$$
d S_{\text {rot }}^{2}=-\left(1+\left(2 B \Omega-\Omega^{2}\right)\left(x^{\prime 2}+y^{\prime 2}\right)\right) d t^{\prime 2}+d x^{\prime 2}+d y^{\prime 2}+d z^{\prime 2}+2(\Omega-B)\left(x^{\prime} d y^{\prime}-y^{\prime} d x^{\prime}\right) d t^{\prime} .
$$

Choosing therefore $\Omega=B$, we find ourselves in a LIF (in the vicinity of the origin). If the two clocks then are rotated as above, would the equality of their ticking rates be restored? At first sight, one might argue as follows: First, as the eigenstates of the hamiltonian (6) have their spins along the $z$ axis, they should not be affected by the rotation of the frame from which we now describe them. Second, the two notions of time (associated with the two, relatively rotating observers) coincide on this axis. Therefore, one might conclude that the effect would persist in the freely falling frame. This would be very puzzling to say the least. However, we must be careful and note that if all we do is change the frame of description, but not make the experimental apparatus (including the detectors) move with the locally inertial frame, then the above mentioned situation would ensue. On the other hand, if we make the experimental apparatus (in particular, the detectors) move together with the locally inertial frame, then the effect will indeed disappear as it should. Right from the outset, we can see that it is not unreasonable, a priori, to expect this, because, in the rotating primed frame, the $S_{z}$-eigenstates are described as

$$
|\hat{z}+\rangle \rightarrow e^{i \Omega t / 2}\left|\hat{z}^{\prime}+\right\rangle, \quad|\hat{z}-\rangle \rightarrow e^{-i \Omega t / 2}\left|\hat{z}^{\prime}-\right\rangle .
$$

The point is that the description of the time evolution of a given state, is different in the rotating and nonrotating frames, despite the fact that, on the $z$ axis, where the particles can be thought to be located for all practical purposes, the two notions of time coincide. As $\left|Q_{a}\right\rangle$ and $\left|Q_{b}\right\rangle$ involve only a single spin eigenstate, the phase factor introduced by a rotation has no observable effect and, therefore, clock $I$ should be insensitive to rotations. On the other hand, $\left|Q_{A}\right\rangle,\left|Q_{B}\right\rangle$ involve both spin eigenstates, each of which transforms with a different phase factor, so that clock $I I$ should be, in principle, affected by rotations.

All this becomes clear if we give a more detailed description of how exactly are the two clocks supposed to operate. Mass oscillations are due to the fact that the experimentally observed particle "flavors", which we denote by $\mid$ top $\rangle$ and $\mid$ bottom $\rangle$, are linear combinations of the mass eigenstates $\left|m_{i}\right\rangle$,

$$
\left.\mid \text { top }\rangle=\frac{1}{\sqrt{2}}\left|m_{1}\right\rangle+\frac{1}{\sqrt{2}}\left|m_{2}\right\rangle, \quad \quad \mid \text { bottom }\right\rangle=-\frac{1}{\sqrt{2}}\left|m_{1}\right\rangle+\frac{1}{\sqrt{2}}\left|m_{2}\right\rangle .
$$

Written in terms of these, the states of clock $I$ become

$$
\begin{aligned}
\left|Q_{a}\right\rangle & =\mid \text { top, } \hat{z}+\rangle \\
\left|Q_{b}\right\rangle & =\mid \text { bottom, } \hat{z}+\rangle,
\end{aligned}
$$




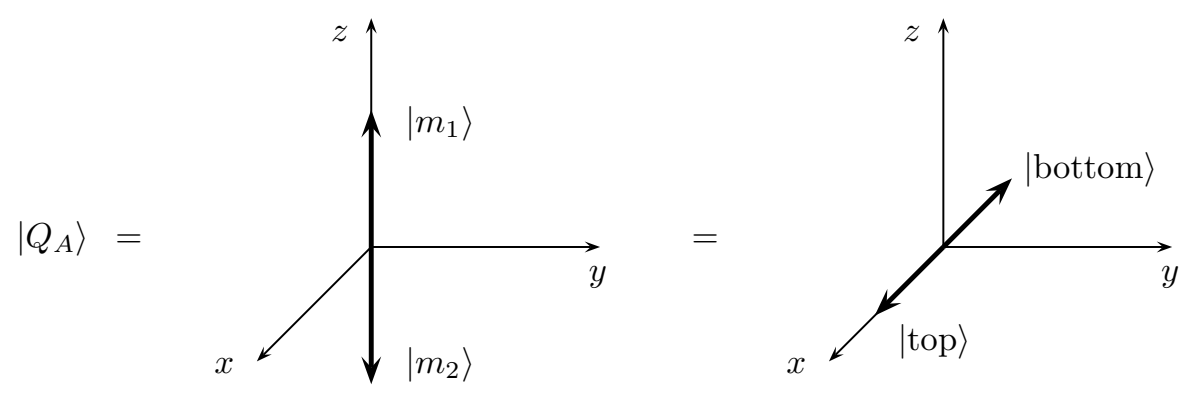

Figure 2: Equivalent ways of describing the state $\left|Q_{A}\right\rangle$. In each case, a sum over the states shown is implied (see Eqs. (3)), (14)). A similar figure can be drawn for $\left|Q_{B}\right\rangle$.

i.e., they correspond to two different flavors with spin along $\hat{z}$. The interesting news is that the states of clock $I I$ become

$$
\begin{aligned}
& \left.\left.\left|Q_{A}\right\rangle=\frac{1}{\sqrt{2}} \mid \text { top, } \hat{x}+\right\rangle+\frac{1}{\sqrt{2}} \mid \text { bottom, } \hat{x}-\right\rangle \\
& \left.\left.\left|Q_{B}\right\rangle=\frac{1}{\sqrt{2}} \mid \text { bottom, } \hat{x}+\right\rangle+\frac{1}{\sqrt{2}} \mid \text { top, } \hat{x}-\right\rangle,
\end{aligned}
$$

i.e., they now involve spins pointing along $\hat{x}$ ! (see Fig. 2). This is the fundamental difference between the two clocks, namely, for clock $I I$, a change in the basis in the mass space affects the direction of the spin as well (which is not true for clock $I$ ). The effect can of course be traced to the fact that while the states of clock $I$ factorize in the two spaces (mass and spin), those of clock $I I$ do not, but rather, involve sums over factorizable states.

Returning to the clock operation, we may now further specify that clock $I$ sends a beam of particles, travelling along the $z$-axis, towards a detector of "top" particles and ticks whenever a maximum counting rate is reached. Clock $I I$ does the same, but first passes the beam through a Stern-Gerlach apparatus which filters out the $|\hat{x}-\rangle$ component $t^{5}$. It is clear from this description that clock $I$ is not affected by rotations around the $z$-axis, as we expected. For clock $I I$, we note that when rotated with angular velocity $\Omega \hat{z}$, the (rotating) Stern-Gerlach apparatus will block the $|\hat{n}-\rangle$-component, where $\hat{n}=(\cos \phi, \sin \phi, 0)$ and $\phi=\Omega t$. It becomes obvious then that, when rotated as above, clock $I I$ actually detects oscillations between the time dependent states $\left|\tilde{Q}_{A}\right\rangle,\left|\tilde{Q}_{B}\right\rangle$, given by

$$
\begin{aligned}
\left|\tilde{Q}_{A}\right\rangle & \left.\left.=\frac{1}{\sqrt{2}} \mid \text { top, } \hat{n}+\right\rangle+\frac{1}{\sqrt{2}} \mid \text { bottom, } \hat{n}-\right\rangle \\
\left|\tilde{Q}_{B}\right\rangle & \left.\left.=\frac{1}{\sqrt{2}} \mid \text { bottom, } \hat{n}+\right\rangle+\frac{1}{\sqrt{2}} \mid \text { top, } \hat{n}-\right\rangle,
\end{aligned}
$$

or, in terms of $\left|Q_{A}\right\rangle,\left|Q_{B}\right\rangle$,

$$
\begin{aligned}
\left|\tilde{Q}_{A}\right\rangle & =\cos \frac{\phi}{2}\left|Q_{A}\right\rangle+i \sin \frac{\phi}{2}\left|Q_{B}\right\rangle \\
\left|\tilde{Q}_{B}\right\rangle & =i \sin \frac{\phi}{2}\left|Q_{A}\right\rangle+\cos \frac{\phi}{2}\left|Q_{B}\right\rangle .
\end{aligned}
$$

\footnotetext{
${ }^{5}$ Notice that the state $\mid$ top, $\left.\hat{x}+\right\rangle$, which is the one detected by the above procedure, only enters in $\left|Q_{A}\right\rangle$ (see (14)), so we can use it as a "tag" for $\left|Q_{A}\right\rangle$ (see also Eq. (17)).
} 
One easily shows that

$$
\left\langle\tilde{Q}_{A} \mid Q_{A}, t\right\rangle=2\left\langle\text { top }, \hat{n}+\mid Q_{A}, t\right\rangle=e^{-i \lambda c^{2}\left(m_{1}+m_{2}\right) t /(2 \hbar)} \cos \left(\frac{\omega_{I I}^{\prime}-\Omega}{2} t\right),
$$

so that the frequency $\Omega$ of the rotation adds to the ticking frequency $\omega_{I I}^{\prime}$ of clock $I I$. When the latter is rotated with $\Omega=B$, the above rotation-induced shift in its frequency exactly cancels the effect of the gravitomagnetic field $\vec{B}$ and the two clocks tick synchronously again. Thus, the effect described in this section is purely geometrical according to the notion of geometricity proposed in (D2).

\section{Discussion}

Before concluding we would like to return to a point briefly mentioned in the introduction: the fact that the equivalence principle is at the foundations of mechanics. We recall that the starting point for the construction of the edifice of classical physics is Newton's three laws, which hold in an inertial frame. How are we supposed, in practice, to find such a frame?. The "frame of the fixed stars", that was considered in Newton's time, would clearly not be an appropriate starting point nowadays. One way to do it is to take three freely moving, non-colinear particles and adjust the motion of our frame so as to ensure that, relative to it, the three particles move according to the law of inertia. At first, this might seem to reduce the law of inertia to a mere definition. However, its content lies in its predictive power regarding the motion of other free particles. If there was no gravitation we could equip ourselves with the required free particles by choosing them electrically neutral (including higher electric or magnetic multipole moments), and ensuring that they were sufficiently distant from other particles so that the nuclear forces could be neglected. However, gravitation exists, so the problem of constructing an inertial frame persists. The EP is what saves the situation: we simply follow the procedure as if there was no gravitation, and the result is a LIF. Moreover, this is the only way to obtain a LIF, unless one such frame is already known and a second one is obtained by moving inertially with respect to the first. In this way, we see that a test of, say, the universality of free fall, using classical objects, should be regarded, if we want to be precise, as a test of the law of inertia.

In the above sense, one is never observing gravitational effects in any local experiment, for any such experimental manifestation simply indicates the failure to construct a LIF. Gravitation manifests itself only in the impossibility to extend our LIF to a global inertial frame, i.e., in its tidal effects this is of course nothing but the general relativistic lesson that gravitation resides in the Riemman curvature tensor. This remark applies, in particular, to the COW experiment, which, in our view, only confirms that the above procedure to construct a LIF, where the laws of mechanics are valid, yields at the same time a frame in which Schroedinger's equation is valid, certainly a highly nontrivial result.

In conclusion, we identify the reason for the sufficiency of (DA) in our analysis so far: none of the experiments that have been carried out to date, as far as we know, attempts to detect gravitational tidal effects using quantum mechanical probes. This is a serious shortcoming of our experimental knowledge in this field, especially if we note that, in accordance to the discussion above, it means that we have not been testing gravitation at all! The point here is not to be critical in any way of the heroic efforts of our experimental colleagues, but just to clarify what indeed has been tested and what still lies ahead. We hope that the challenge of detecting gravitational tidal effects on quantum systems will soon be undertaken.

\section{Acknowledgments}

The authors would like to acknowledge partial support from CONACyT projects 32307-E (C. C.), 32272-E (D. S.) and DGAPA-UNAM projects IN 119792 (C. C.), IN 121298 (D. S.).

\footnotetext{
${ }^{6}\left|Q_{A}, t\right\rangle$ is the time-evolved ket that, at $t=0$, coincides with $\left|Q_{A}\right\rangle$.
} 


\section{References}

[1] D. Ahluwalia. On a New Non-Geometric Element in Gravity. Gen. Rel. and Grav., 29:1491, 1997.

[2] G. Amelino-Camelia. Are We at the Dawn of Quantum Gravity Phenomenology? Lect. Notes Phys., 541:1, 2000.

[3] R. Colella, A. W. Overhauser, and S. Werner. Observation of Gravitationally Induced Quantum Interference. Phys. Rev. Lett., 34:1472, 1975.

[4] V. V. Nesvizhevsky et al. Quantum States of Neutrons in the Earth's Gravitational Field. Nature, 415:297-299, 2002.

[5] T. Jacobson. Thermodynamics of Spacetime: the Einstein Equation of State. Phys. Rev. Lett., $75: 1260-1263,1995$.

[6] J. J. Sakurai. Modern Quantum Mechanics (Revised Edition). Addison-Wesley, 1994.

[7] R. M. Wald. General Relativity. University of Chicago Press, 1984. 